\title{
Análise por ondaletas sobre níveis de rios submetidos à influência de maré
}

\author{
Analysis of the level of rivers under tidal influence using wavelets
}

\section{Antonio Eliseu Holdefer ${ }^{1}$ e Dirceu Luis Severo ${ }^{2}$}

${ }^{1}$ Holdefer monitoramento industrial e ambiental

holdefer@gmail.com

${ }^{2}$ Departamento de Física, Universidade Regional de Blumenau (FURB), Blumenau, SC- Brasil.

dirceuluis@gmail.com

Recebido: 11/05/14 - Revisado: 07/08/14 - Aceito: 30/10/14

\begin{abstract}
RESUMO
Certos cursos de água tais como ribeirões, próximos a regiões costeiras e que são afuentes de rios maiores, que por sua vez. deságuam no mar, podem sofrer influência direta da ação de refluxos de maré. O fenômeno pode se mostrar desprezível em condições de maior vazãa, porém o mesmo pode se tornar perceptivel em condições de baixa vazão. Erros de cálculo de vazão em situações de niveis baixos podem ser consideráveis se um sistema de suavização de-noising adequado não for utilizado para eliminar os efeitos da maré. Muitas vezes, examinando-se a série histórica hidrológica e usando-se a análise de Fourier padrão no dominio de tempo-frequência, pode não ser possivel identificar de forma conclusiva onde se encontram as fontes de ruído (maré). No entanto, uma nova perspectiva pode ser obtida por meio da transformada wavelet ou ondaleta, em português, usada para analisar a série temporal no domínio de tempo-escala. Usando-se essa abordagem, um estudo de caso que envolve uma série hidrológica de nível observada no Ribeirão da Velha em Blumenau-SC é apresentado, no qual se aproveitou a instalação de uma nova rede telemétrica de medição de nivel, com aquisişão de dados em intervalos de 5 minutos, tornando-se possivel a medição da maré de maneira precisa devido à alta resolução temporal. Tal análise rendeu provas conclusivas da influência das marés no local de medição de nivel em periodos de baixo fluxo. Após a confirmação que a existência de futuações de pequena amplitude são devidas à maré, a série de niveis de Ribeirão da Velha foi submetida a um procedimento de-noising, apropriado para obter uma série suavizada, que posteriormente foi submetida à análise de wavelet, evidenciando o desaparecimento do efeito da maré. Em seguida, provou-se, através de análise de coerência de potência e fase, que o efeito da maré no Ribeirão da Velha provém do Rio Itajaí-açú, no qual o primeiro deságua. Paralelamente, também se mostrou plausivel o cálculo do atraso, com a posterior dedução da velocidade de deslocamento da maré através do ribeirão.
\end{abstract}

Palavras Chave: Espectro de potência de Fourier. Hidrologia. Maré. Transformada wavelet. De-noising. Espectro de potência wavelet.

\begin{abstract}
Certain water courses such as large streams close to coastal regions and that are tributaries of larger rivers which run into the sea may be directly influenced by tidal ebb. The phenomenon may be negligible when there are heavier flow conditions, but it may become perceptible at low flow. Errors in calculating flow when levels are low may be considerable if an adequate smooth de-noising system.system is not used to eliminate the effects of the tide. Often, when one looks at the hydrological historical series and using the standard Fourier analysis in the time-frequency domain, it may not be possible to identify conclusively where the sources of noise are (tide). However, a new perspective can be obtained by wavelet transformation which is used to analyze the time series in the time-scale domain. Using this approach, a case study that involves a hydrological series of levels observed in the Ribeirão da Velha Blumenau-SC is presented, in which the installation of a new telemetric network for level measurement was used, with the acquisition of data at 5-minute intervals, making it possible to measure the tide precisely due to the high time resolution. This analysis provided conclusive proof of the influence of tides at the measurement site during low flow periods. After the confirmation that the existence of small range oscillations is due to the tide, the Ribeiro da Velha series of levels was submitted to a de-noising procedure, appropriate to obtain a smoother series which was later submitted to wavelet analysis, showing the disappearance of the tide effect. Then, by analysis of the consistency of power and phase, it was proved that the effect of the tide on Ribeirão da Velha comes from the Itajai-Açu river, into which the former runs. At the same time it was found plausible to calculate the lag, with a later reduction of the tide displacement velocity through the stream
\end{abstract}

Keywords: Fourier power spectrum. Hidrology. Tide. Wavelet transformation. De-noising. Wavelet power spectrum. 


\section{INTRODUÇÃO}

Atualmente, o estudo de sinais aleatórios não estacionários exige a utilização de técnicas matemáticas robustas de análise para poder observar, caracterizar e compreender os fenômenos que estão atuando. Exemplos clássicos de dados que apresentam variabilidade complexa são aqueles relacionados à observação de variáveis atmosféricas e do meio ambiente (velocidade de vento, temperatura do ar, crescimento de biomassa, nível de rios, etc.) (BARBOSA et al., 2005).

A análise por wavelets é um grande avanço nos métodos de análise de dados dos últimos trinta anos. Sua origem está na geofísica, por Morlet, para analisar sinais sísmicos (Morlet et al. (1982a,b)), sendo que posteriormente evoluiu a partir de campos diversos, tais como processamento de sinais, física e matemática. A aplicação da transformada wavelet na análise de dados temporais, especialmente aqueles com características não estacionárias, foi muito bem sucedida (NASON; SACHS, 1999; PERCIVAL; WALDEN, 2000). A propriedade de localizar no tempo ou espaço, bem como em escala (potência) ou frequência, fornecer o mapa de tempo-escala de um sinal, permite a extração de características que podem variar no tempo. Isso faz da transformada wavelet uma ferramenta ideal para a análise de sinais de natureza transiente ou não estacionária.

A literatura sobre a aplicação da transformada wavelet na área de hidrologia e engenharia de recursos hídricos é omissa e relativamente escassa. Jay e Flinchem $(1995,1997,1999)$ demonstraram a interação da flutuação no fluxo de rios com marés barotrópicas usando análise wavelet. Smith et al. (1998) foram capazes de caracterizar cursos de água de diferentes regiões climáticas nos Estados Unidos usando a transformada wavelet. Aplicações na área de meteorologia também são importantes quando intimamente ligadas à hidrologia. Um excelente exemplo dessa relação é o documento apresentado por Torrence e Compo (1998). Gan (2001) demonstrou a evidência de que as secas nas pradarias canadenses estão relacionadas com a circulação de correntes marítimas padrão no Oceano Pacífico utilizando uma análise por wavelets. Lim e Lye (2004) demonstraram, através da análise por wavelets, que o efeito da maré em cursos de água durante períodos de baixa vazão podem afetar de forma significativa o cálculo da curva chave. Muitos aspectos de hidrologia, de uma forma ou de outra, envolvem a análise de uma série temporal hidrológica. Assim, se houver qualquer melhoria significativa na técnica de análise de séries temporais, pode haver um desenvolvimento significativo sobre o entendimento de "processos hidrológicos" de uma maneira geral.

Não é possível verificar a presença das marés quando a flutuação do nível dos rios é devida a outros processos dinâmicos, tal como o efeito do escoamento local devido a eventos localizados de chuva. Se a presença do efeito das marés é comprovada, a série em períodos de baixa vazão pode ser suavizada usando um método de-noising robusto (suavização) cancelando o efeito da maré na série de dados em questão.

Apesar do Ribeirão da Velha ser um pequeno tributário que desemboca após $2.56 \mathrm{~km}$ (ponto de medição) no rio Itajaí-Açú, e o mesmo estar a uma distância de 68.9 km do mar, técnicos que frequentemente aferem o local informam que o nível do ribeirão pode ser afetado pela maré durante períodos de baixa vazão. É de fato difícil confirmar a validade do argumento baseando-se em uma verificação casual dos valores flutuantes de nível. Esse tipo de problema é bastante comum em lugares onde o efeito da maré atinge grandes distâncias continente adentro.

A análise do efeito da maré no presente ribeirão se tornou possível devido à instalação, em 2009, de uma estação telemétrica automática de coleta de nível, permitindo aquisições quase em tempo real, com intervalos entre coletas de 5 min.

\section{DADOS E METODOLOGIA}

Os dados utilizados neste estudo foram obtidos de duas estações telemétricas de monitoramento de nível, localizadas na cidade de Blumenau-SC, uma instalada no Ribeirão da Velha e a outra no rio Itajaí-Açú, tal como mostrado na Figura 1. A estação

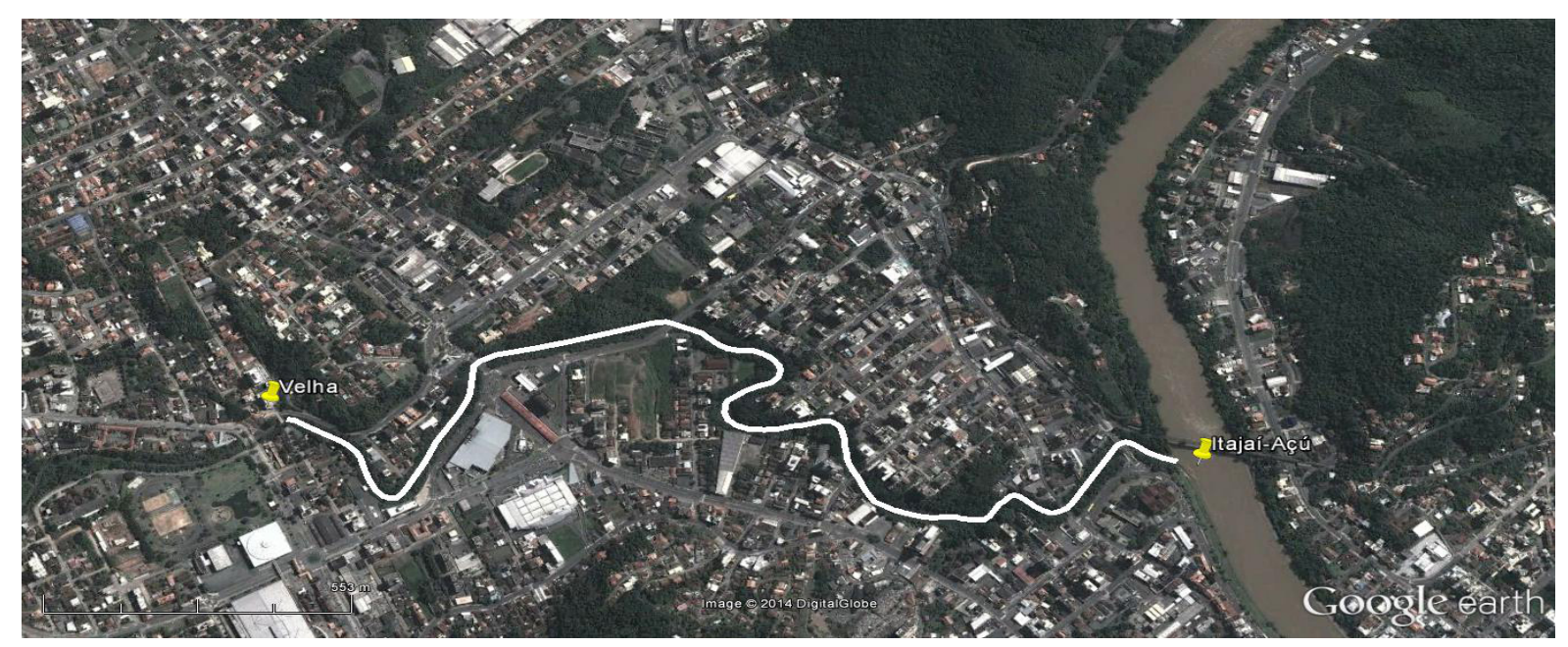

Figura 1 - Mapa de Blumenau, detalhe em branco do Ribeirão da Velha, e pontos de medição no Ribeirão da Velha (Bairro da Velha em Blumenau) e no rio Itajaí-Açú (Ponto em que o Ribeirão da Velha desemboca no rio Itajái-Açú) 


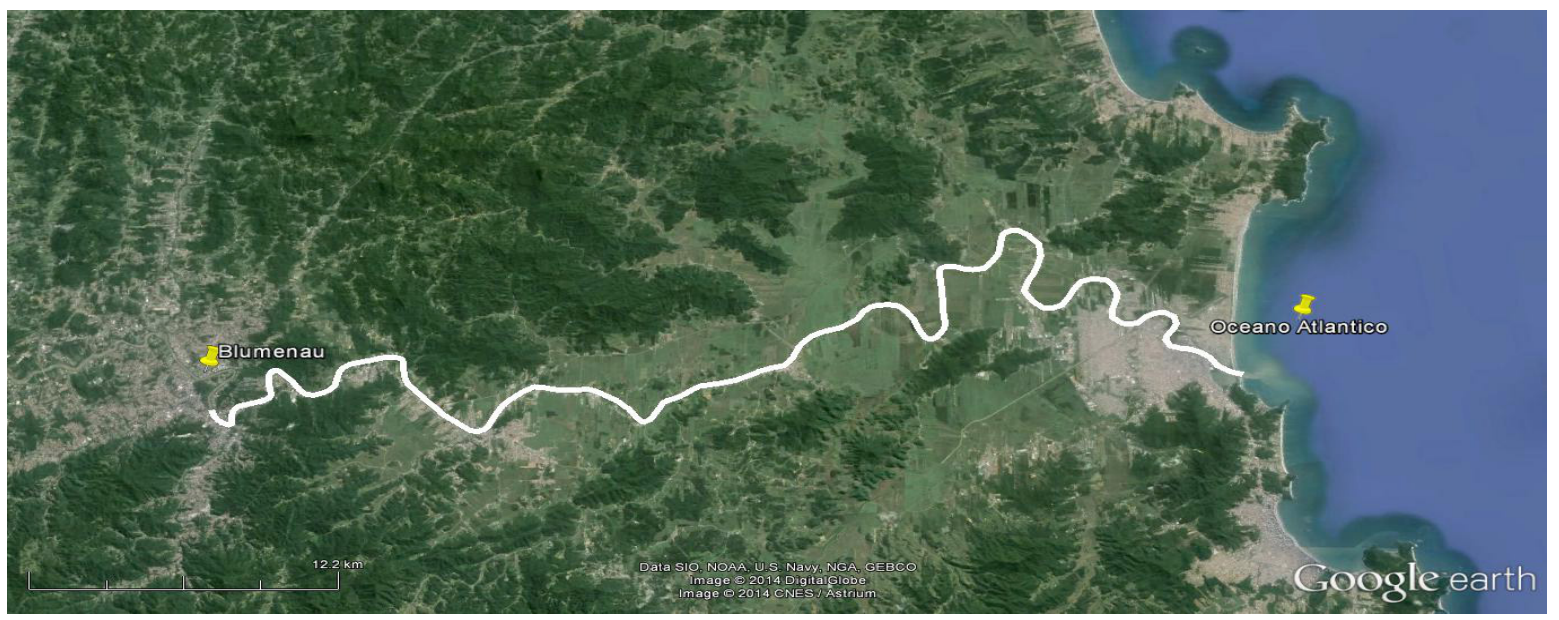

Figura 2 - Mapa da posição relativa de Blumenau em relaçao ao Oceano, com o rio Itajaí-Açú destacado em branco

instalada no rio Itajaí-Açú se encontra próxima da desembocadura do Ribeirão da Velha. O curso do Ribeirão da Velha, entre o ponto de medição e o rio Itajaí-Açú, é mostrado em branco na Figura 1, sendo que a distância entre as duas estações é de $2.56 \mathrm{~km}$. A Figura 2 mostra a posição relativa da Blumenau até o oceano atlântico. A distância percorrida pelo rio Itajaí-Açú do ponto de medição em Blumenau até a desembocadura no oceano Atlântico é de $68.9 \mathrm{~km}$. O curso do rio Itajaí-Açú até o oceano é mostrado em branco na Figura 2.

A estação telemétrica instalada no Ribeirão da Velha adquire os dados de nível a cada 5 minutos, e utiliza um sensor de pressão submerso com compensação barométrica para a realização das medições. Por outro lado, a estação telemétrica no rio Itajaí-Açú utiliza um sensor ultrassônico (radar) instalado acima do nível do rio e adquire os dados em intervalos de 15 minutos.

Para a realização da análise multirresolução, a transformada wavelet faz uso de uma função base, chamada wavelet mãe. A wavelet mãe é uma onda de pequeno comprimento que cresce e decai em um período limitado (PERCIVAL; WALDEN, 2000; TORRENCE; COMPO, 1998). A wavelet mãe é o ponto crucial da análise wavelet, pois de acordo com a escolha da onda a ser utilizada, a transformada irá identificar ou desprezar determinadas informações durante o processo de criação do gráfico do espectro de potência wavelet.

Para que a transformada wavelet funcione de maneira adequada é necessário que a wavelet mãe $\psi(\mathrm{t})$ utilizada satisfaça as seguintes restrições:

$$
\int_{-\infty}^{+\infty} \Psi(t) d t=0
$$

e

$$
\int_{-\infty}^{+\infty} \Psi^{2}(t) d t=1
$$

Esta wavelet mãe pode ser usada para gerar uma família completa de wavelets por meio da translação e escalonamento, tal como mostrado por:

$$
\Psi_{(a, b)}(t)=\frac{1}{\sqrt{a}} \Psi\left(\frac{t-b}{a}\right), a>0
$$

onde $b$ é o parâmetro de translação e $a$ é o parâmetro de escalonamento.

A transformada wavelet de uma função $f(t)$ é definida por meio da integral

$$
W_{(a, b)}=\int_{-\infty}^{+\infty} f(t) \Psi_{(a, b)}(t) d t
$$

Ao mudar-se o parâmetro $t$ tem-se o efeito de analisar a função $f(t)$ em torno de diferentes pontos no tempo. Quando o fator de escala $a$ aumenta tem o efeito de tornar a wavelet mais espalhada, o que leva em consideração somente oscilações de períodos maiores da função $f(t)$ e vice-versa. Dessa forma a transformada wavelet provê uma janela tempo-escala flexível que se estreita quando focada em características de alta frequência e que se alarga quando focada em características de baixa frequência.

O espectro de potência wavelet é extraído da transformada wavelet, e é definido por:

$$
P_{(a, b)}=\left|W_{(a, b)}\right|^{2} .
$$

O módulo da função é aqui utilizado, pois geralmente a função $\Psi_{(a, b)}(t)$ é complexa, e consequentemente também a função $W_{(a, b)}$.

Para a geração do gráfico de potência wavelet definem-se dois vetores. Um contendo a sequência de valores para $a$, que corresponderão aos valores de escala que deverão ser aplicados à função $W_{(a, b)}$ e que corresponderão ao eixo vertical do gráfico, e o outro contendo a sequência de valores para $b$, que corresponderão aos valores do deslocamento que deverão ser aplicados à função $W_{(a, b)}$ e que corresponderão ao eixo horizontal do gráfico.

Exemplos típicos de wavelets mães são as de Morlet, Haar e Daubechies (NASON; SACHS, 1999; KUMAR; FOUFOULA-GEORGIOU, 1997).

A função wavelet mãe escolhida para este trabalho foi a Morlet, que por ser complexa fornece informações a respeito da energia (amplitude) e fase (parte imaginária), segundo Weng e Lau (1994). Esta função possui a seguinte forma:

$$
\Psi(t)=\pi^{-1 / 4} e^{i \omega_{0} t} e^{-t^{2} / 2}
$$


Essa função também é a mais adequada à captação de sinais atmosféricos e hidrológicos com características simétricas e assimétricas de maneira contínua ao longo do tempo (DAUBECHIES, 1992; WENG; LAU, 1994; TORRENCE; COMPO, 1998).

Em algumas aplicações, torna-se interessante suavizar o sinal, atenuando as frequências mais altas, por um processo chamado "suavização" ou de-noising (em Inglês).

Em geral, um sinal suavizado $s(t)$ pode ser recuperado a partir de um sinal $y(t)$ pela expressão:

$$
s(t)=y(t)-\sigma x(t) \quad,
$$

onde $\mathrm{x}(\mathrm{t})$ representa as oscilações em alta frequência e $\sigma$ é o nível de tais oscilações. O método de suavização wavelet é uma estimativa da função $s(t)$ usando-se uma base ortogonal, utilizando-se a transformada wavelet, dada pela equação 4. A principal razão de se usar o método de-noising utilizando-se wavelets é que elas permitem a atenuação no sinal de alta frequência sem a mudança de fase (atraso ou avanço), mantendo as características temporais do sinal. O mesmo não ocorre com métodos convencionais, tais como filtro média-móvel ou filtro passa-baixa, que sempre adicionam fase ao sinal, tal como verificado em (DONOHO; JOHNSTONE, 1994; COIFMAN; DONOHO, 1995).

Há várias questões a serem abordadas na suavização wavelet, entre as quais as escolhas da wavelet mãe, os níveis de decomposição, e os métodos de limiarização. As descrições dessas opções são encontradas em artigos como o de Donoho e Johnstone (1994, 1995), entre outros. No entanto, alguns julgamentos heurísticos ainda são necessários na escolha da melhor combinação.

\section{RESULTADOS}

O problema do efeito da maré no Ribeirão da Velha torna-se de interesse, pois ajuda a corrigir possíveis erros de cálculo de vazão durante períodos de baixa vazão do rio, os quais podem ser corrigidos pelo processo de "suavização" do sinal. O entendimento do efeito da maré no ribeirão também ajuda numa melhor compreensão da dinâmica existente entre o mesmo e o rio Itajaí-Açú.

A investigação do problema utilizando-se métodos tradicionais envolveria a análise por utilização da transformada de Fourier, a qual provê alguma compreensão a respeito da ocorrência da maré. Entretanto, como será mostrado posteriormente, a análise via transformada wavelet oferece uma perspectiva de análise totalmente diferente que conduz a conclusões muito mais sólidas e objetivas.

A Figura 3 mostra o gráfico do nível do Ribeirão da Velha, com amostragem de 5 em 5 minutos e duração de 168 dias, começando em $1^{\circ}$ de novembro de 2012 . Pode se ver algumas flutuações de alta frequência durante os períodos de baixos níveis e, consequentemente, baixa vazão. Tais oscilações são mostradas em maior detalhe na Figura 4, na qual foi selecionado um segmento do gráfico durante um período de baixa vazão.

A Figura 5 mostra o espectro de potência de Fourier para valores horários, para o mesmo período de 168 dias. O espectro mostra dois picos distintos, nos períodos de $23.92 \mathrm{e}$ $11.98 \mathrm{~h}$, ou frequências de $0.0418 \mathrm{~h}^{-1}$ e $0.08346 \mathrm{~h}^{-1}$, respectivamente. O fenômeno da maré é causado pelo campo gravitacional da Lua, o qual tem um período em torno de $12.5 \mathrm{~h}$. Outros períodos também podem ser observados, como o de $23.92 \mathrm{~h}$, que pode ser causado por outros efeitos lunares ou solares. Essas periodicidades específicas certamente correspondem a assinaturas rastreáveis das marés de desigualdades diurnas, que dominam as águas costeiras da região sul do Brasil (TRUCCOLO; SCHETTINI, 1999).

Apesar da coincidência na proximidade dos valores, a presença da maré no nível do Ribeirão da Velha durante períodos de baixa vazão ainda é apenas um indício, pois ainda não se consegue correlacionar essas oscilações com os períodos de baixa vazão do rio. Desde que nenhuma outra informação pode ser retirada do gráfico do espectro de potência de níveis, nenhuma conclusão firme pode ser feita.

A transformada wavelet é uma ferramenta analítica poderosa, que está cada vez mais sendo usada como uma alternativa à análise de Fourier. Naturalmente, se espera que o uso de tal transformada possa gerar uma melhor perspectiva de

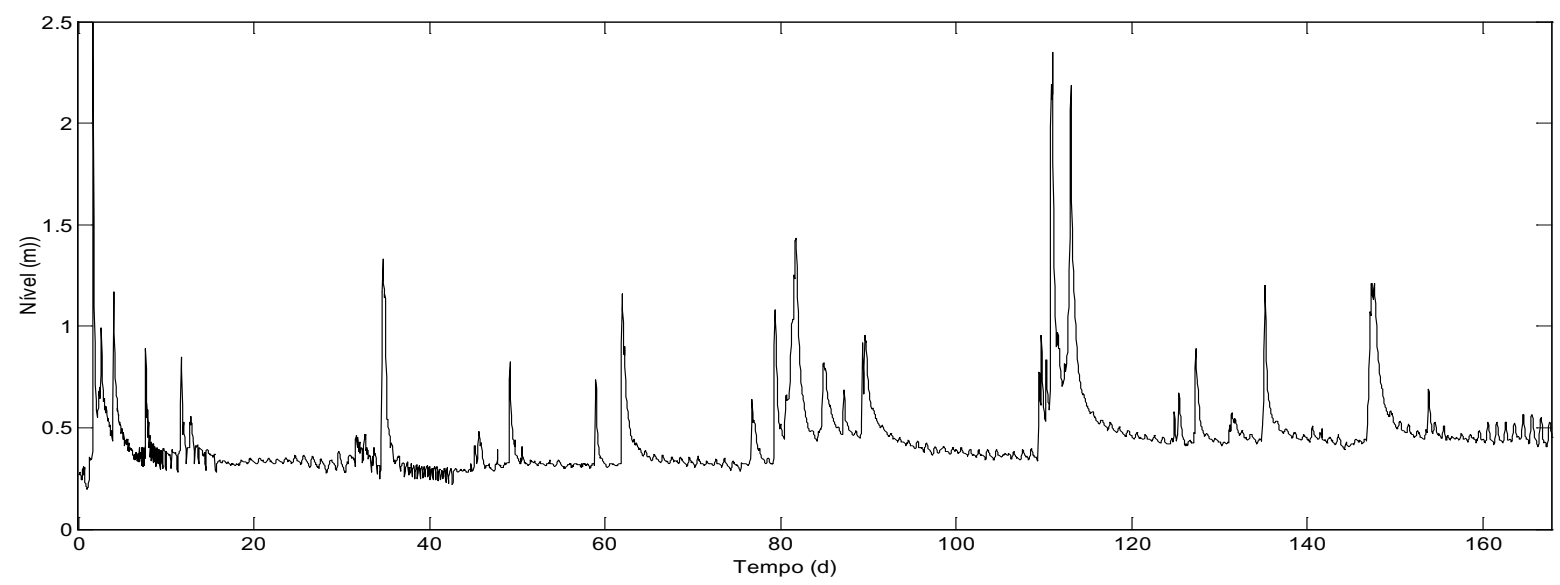

Figura 3 - Nível do Ribeirão da Velha, no ponto de medição, para um período de 168 dias 


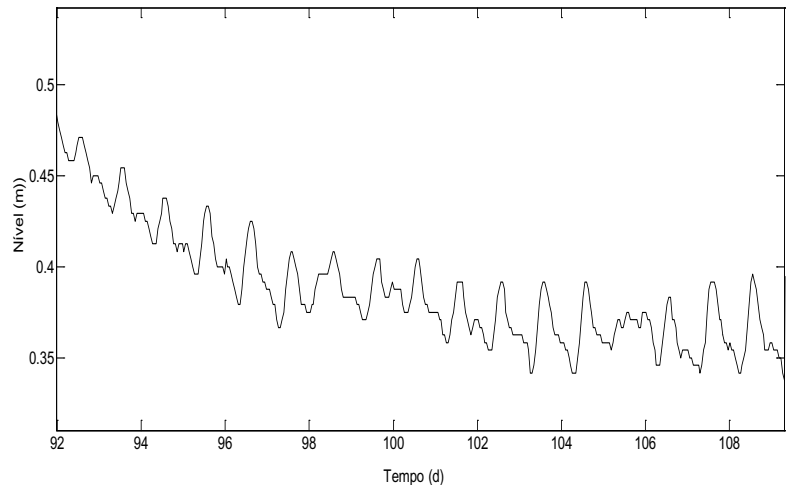

Figura 4 - Nível do ribeirão da velha, segmento de baixa vazão em detalhe

compreensão do fenômeno em análise.

$\mathrm{Na}$ Figura 6 o espectro de potência wavelet (equação 5), foi obtido através do uso da transformada wavelet (equação 4). Nesse gráfico de potência wavelet, que corresponde a maior parte da Figura 6, o eixo $x$ corresponde ao tempo em dias, da função analisada, que nesse caso é o nível do Ribeirão da Velha e o eixo $y$ corresponde ao período de oscilação $(1 / f)$. A partir de uma inspeção no gráfico do espectro de potência, não é difícil perceber a ocorrência de "manchas" distintas de relativa potência em torno dos períodos de 12 e 24 h, evidenciadas preliminarmente pela análise de Fourier (fig. 5). Uma seta e um círculo foram colocados em um ponto de baixa vazão no mapa wavelets, de forma a indicar que a ocorrência dessas "manchas" corresponde aos períodos de baixa vazão do Ribeirão da Velha, o qual pode ser evidenciado no gráfico de nível.

Ao se tomar o espectro de potência wavelet e a partir deste efetuar o somatório de todos os espectros de potência wavelet locais, se obtém um espectro de potência wavelet global. Este gráfico é mostrado na parte inferior direita da Figura 6.

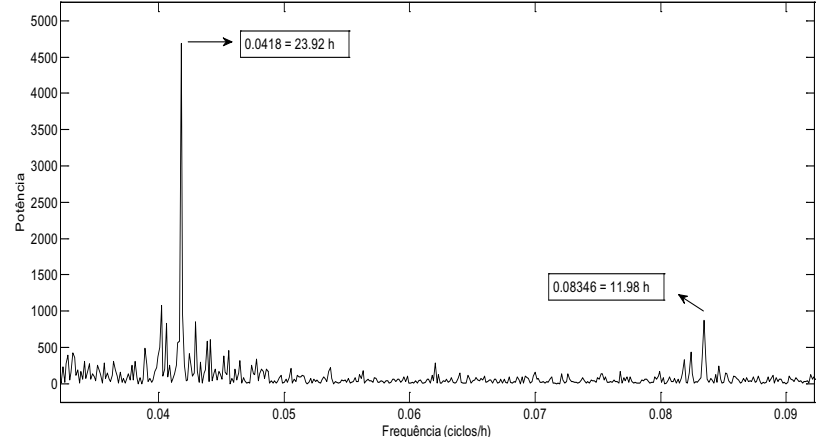

Figura 5 - Espectro de Fourier para a série de dados de nível do ribeirão da velha

Após a verificação do gráfico percebe-se a existência de dois picos suaves em torno do período de 12 e 24 h, porém não proeminentes, o que mostra que a componente de frequência mais elevada atribuída às marés não é dominante durante todo o período da série.

Com isso pode-se concluir que a análise via transformada de wavelet adicionou uma dimensão à informação que havia no sentido de "tempo-escala", a qual não pôde ser obtida via análise de Fourier. Assim, fica claro que as flutuações de nível que ocorrem em períodos de baixa vazão têm uma periodicidade em torno de 12 e $24 \mathrm{~h}$.

Os dados de nível do Ribeirão da Velha foram posteriormente sujeitos a um processo de de-noising, de maneira a extrair a componente de alta frequência (maré).

Como foi verificado anteriormente, o efeito da maré no Ribeirão da Velha é somente observável em períodos de baixa vazão. Portanto, ao se realizar o processo de "de-noising", suporse-á que existe um nível máximo $A_{\text {max }}$ para o local, a partir do qual o efeito da maré não é mais observável. $A_{\text {max }}$ será utilizado como limite para o processo de suavização da série de dados.

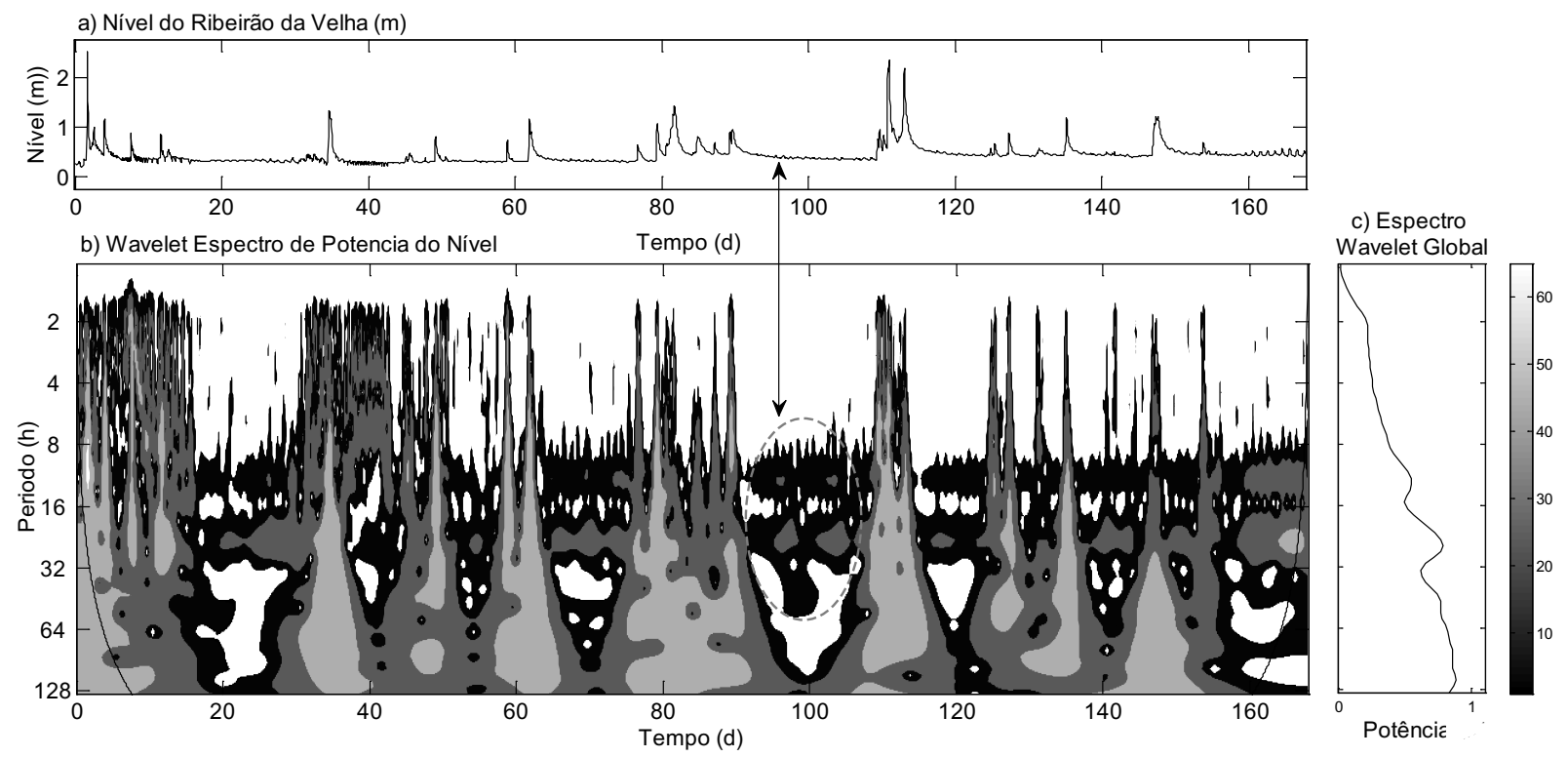

Figura 6 - Análise wavelet do nível do ribeirão da velha (a) série temporal de nível, (b) Espectro de Potência Wavelet e (c) Espectro de potência global 


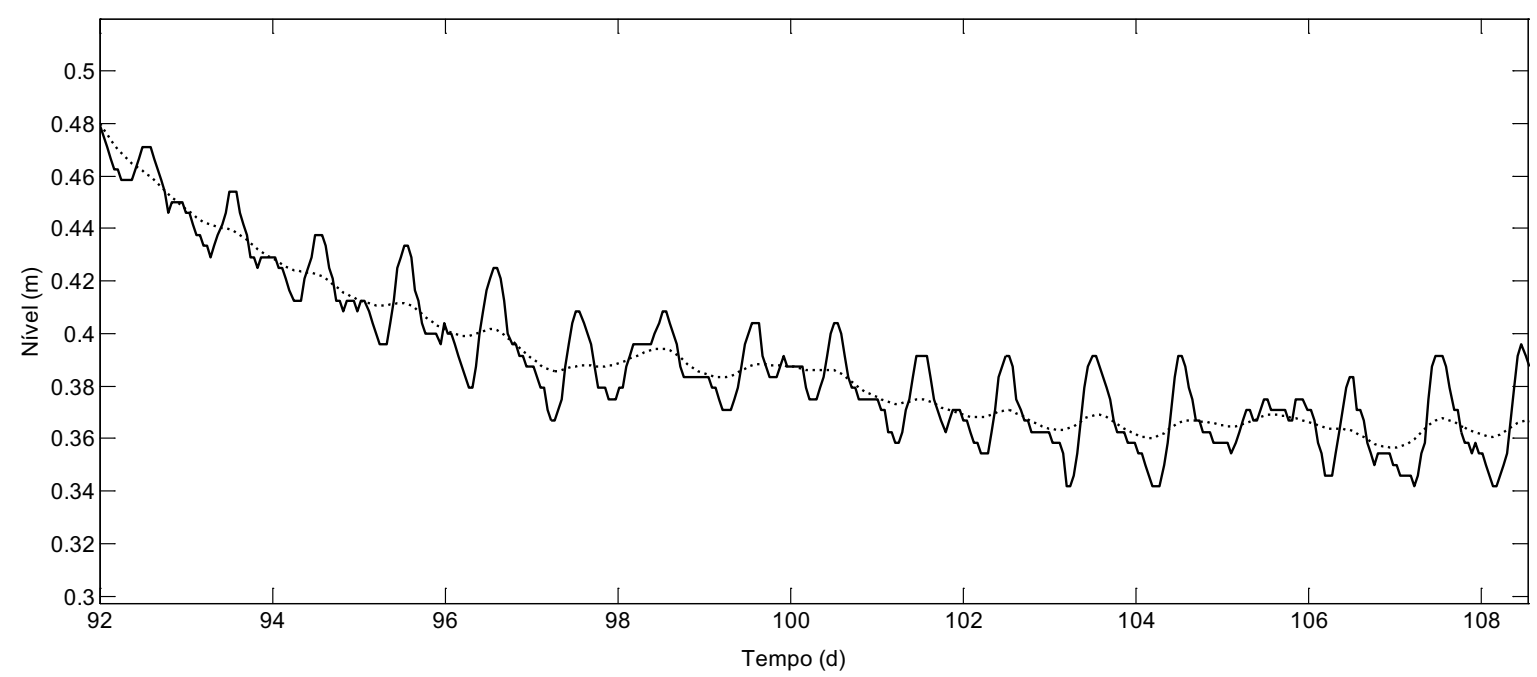

Figura 7 - Detalhe do nível (linha contínua) do Ribeirão da Velha com o sinal suavizado (linha pontilhada)

Para esse caso é sugerido o seguinte algoritmo:

1. Adaptar uma combinação satisfatória de opção de suavização wavelet da série de dados com base em $A_{\text {max }}$;

2. Realizar o processo de de-noising sobre a série de nível da água $y(t)$ e obter uma série $s(t)$ suavizada;

3. Para $s(t)<A \max , s(t)$ é transformado em uma série suavizada z $(t)$, e para $s(t)>A$ max, o segmento de série original $y(t)$ é atribuído a z $(t)$;

4. Verificar a continuidade e suavidade na transição da série de dados ao redor de $A$ max.

5. Ajustar Amax e repetir os passos de 2 a 4 até que os critérios de continuidade e suavidade sejam satisfeitos.

Todos os passos indicados nos itens de 1 a 5 podem ser realizados por meio do pacote de ferramentas Wavemenu, presente no software Matlab. Várias combinações envolvendo diferentes opções de wavelets, níveis de decomposição e de limiar estão disponíveis e podem ser considerados.

Uma breve revisão nos valores da série de dados de nível do Ribeirão da Velha mostra que, em média, durante períodos de baixa vazão, a taxa de variação do nível é $1.33 \mathrm{~cm} /$ hora, sendo que os valores mais altos em épocas de enchente foram de até $0.5 \mathrm{~m} /$ hora. Estes valores físicos podem ser utilizados para eliminar algumas combinações pobres sobre o tipo de wavelet e do nível de decomposição a ser escolhido. Entretanto, será uma decisão heurística escolher qual é a melhor solução entre as disponíveis.

Neste estudo usou-se o processo de suavização utilizando wavelets Daubechies-4, que mostrou o melhor resultado de atenuação dentre as wavelets testadas através do uso do pacote de ferramentas Wavemenu do software Matlab. O resultado da suavização é mostrado na Figura 7 , onde se percebe que a oscilação de alta frequência (maré) foi bastante atenuada.

Para confirmar a eficácia do processo de de-noising, foi gerado o espectro de potência wavelet para o sinal suavizado (Figura 8). Em comparação com a Figura 6 (sinal original), verifica-se que as regiões correspondentes às oscilações periódicas em torno de 12 e $24 \mathrm{~h}$ foram removidas. Para melhor visualização, em uma dessas regiões foi colocado um círculo pontilhado. Esse fato também pode ser confirmado após visualização do espectro wavelet global (Figura 8c), onde se percebe que os dois picos em torno do período de 12 e $24 \mathrm{~h}$ foram retirados, confirmando-se assim que o efeito da das oscilações de alta frequência (maré) no espectro de potências foi bastante atenuado.

Outra análise, para verificar se a ocorrência da maré no Ribeirão da Velha é devida ao rio Itajaí-Açú, baseou-se na correlação entre a maré que age sobre o rio e sobre o ribeirão. A existência da maré no ribeirão da Velha foi evidenciada nos parágrafos anteriores.

O efeito da maré no rio Itajaí-Açú, que desemboca no mar é muito mais evidente visualmente que no ribeirão da Velha, tal efeito pode ser evidenciado pela Figura 9, na qual notam-se picos de maré aproximadamente a cada $12 \mathrm{~h}$.

Para a análise da correlação entre a maré do Ribeirão da Velha e do rio Itajaí-Açú, escolheu-se um período de 5 dias, do dia 04/09/2013 ao 09/09/2013, no qual não houve a ocorrência de chuvas, mantendo os níveis de vazão baixos, aumentando-se assim a influência da maré nos mesmos e facilitando a análise dos dados.

$\mathrm{Na}$ Figura 10 aplicou-se a transformada wavelet para cada um dos dois sinais, e, por esses gráficos, tem-se uma ideia inicial do intervalo de tempo em que ambos os sinais "carregam" mais potência. Pela paleta de tons de cinza percebe-se que as maiores potências são representadas pelas cores mais claras. Portanto verifica-se, no caso do rio Itajaí-Açú, um valor significativo ao redor de $12 \mathrm{~h}$ de intervalo, e no caso do Ribeirão da Velha encontram-se potências significativas em torno dos intervalos de 12 e $24 \mathrm{~h}$.

Apesar de essa informação ser significativa, ela não nos confirma que existe correlação entre os dois sinais.

Para a referida análise de correlação utilizou-se as ferramentas XWT (Cross Wavelet Transform), ou transformada cruzada Wavelet, e a WTC (Wavelet Coherence), ou coerência Wavelet, que são encontradas no software Matlab, tal como sugerido por (GRINSTED et al. 2004).

Com a primeira ferramenta (XWT) se detectam regiões no tempo e na frequência onde ambos os sinais mostram combinações de potência em comum. A segunda ferramenta (WTC) coloca em evidência regiões no tempo e na frequência 


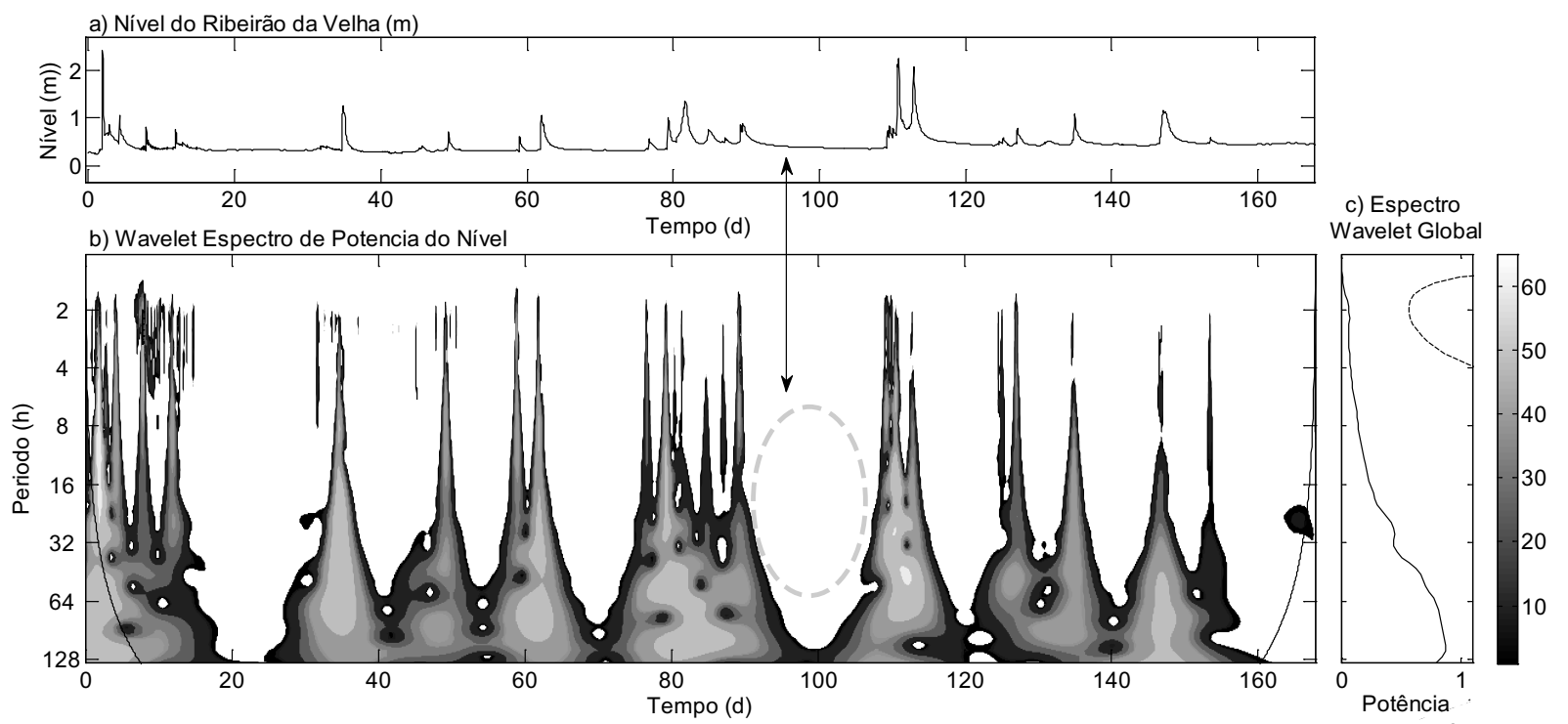

Figura 8 - Análise wavelet do nível do ribeirão da velha após suavização do sinal (a) série temporal de nível, (b) espectro de Potência Wavelet e (c) espectro de potência global
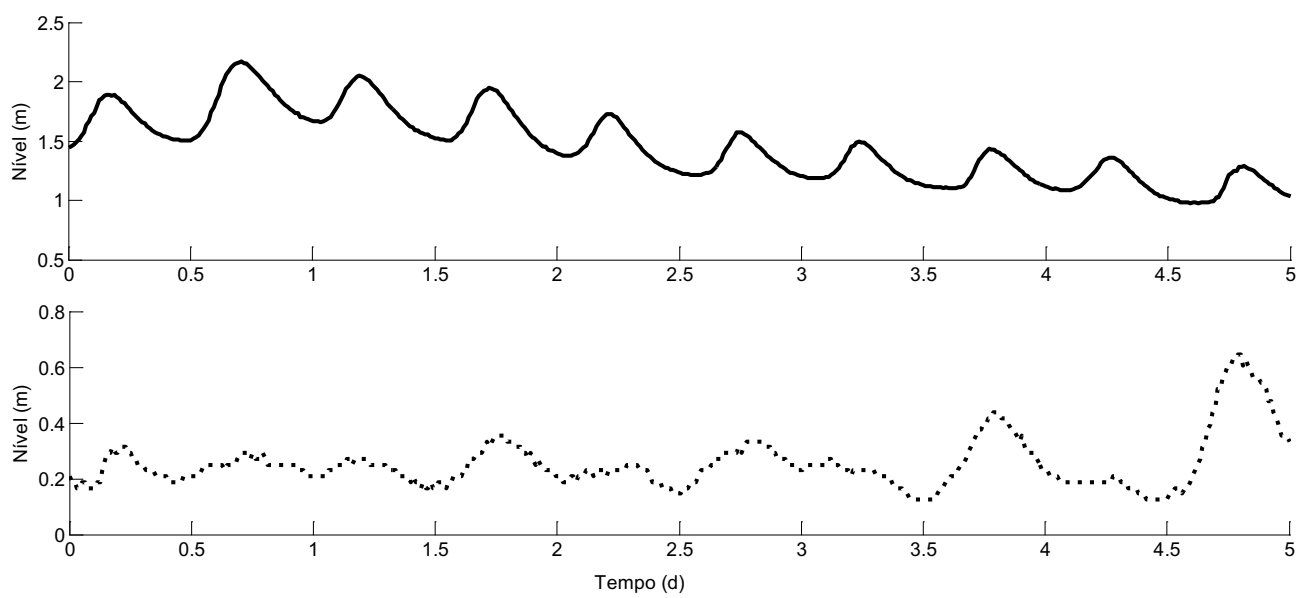

Figura 9 - Nível do rio Itajaí-Açú (linha contínua) e do Ribeirão da Velha (linha pontilhada) para o período de 04/09/2013 a 09/09/2013

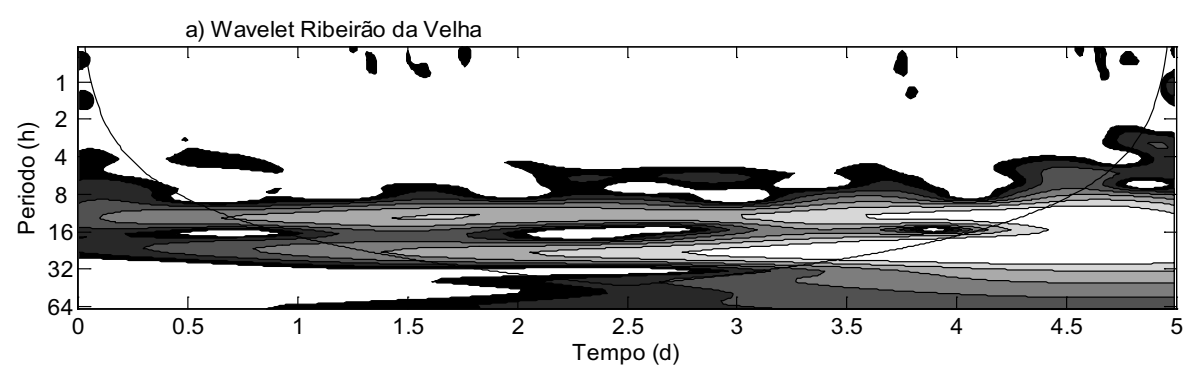

b) Espectro Wavelet Global
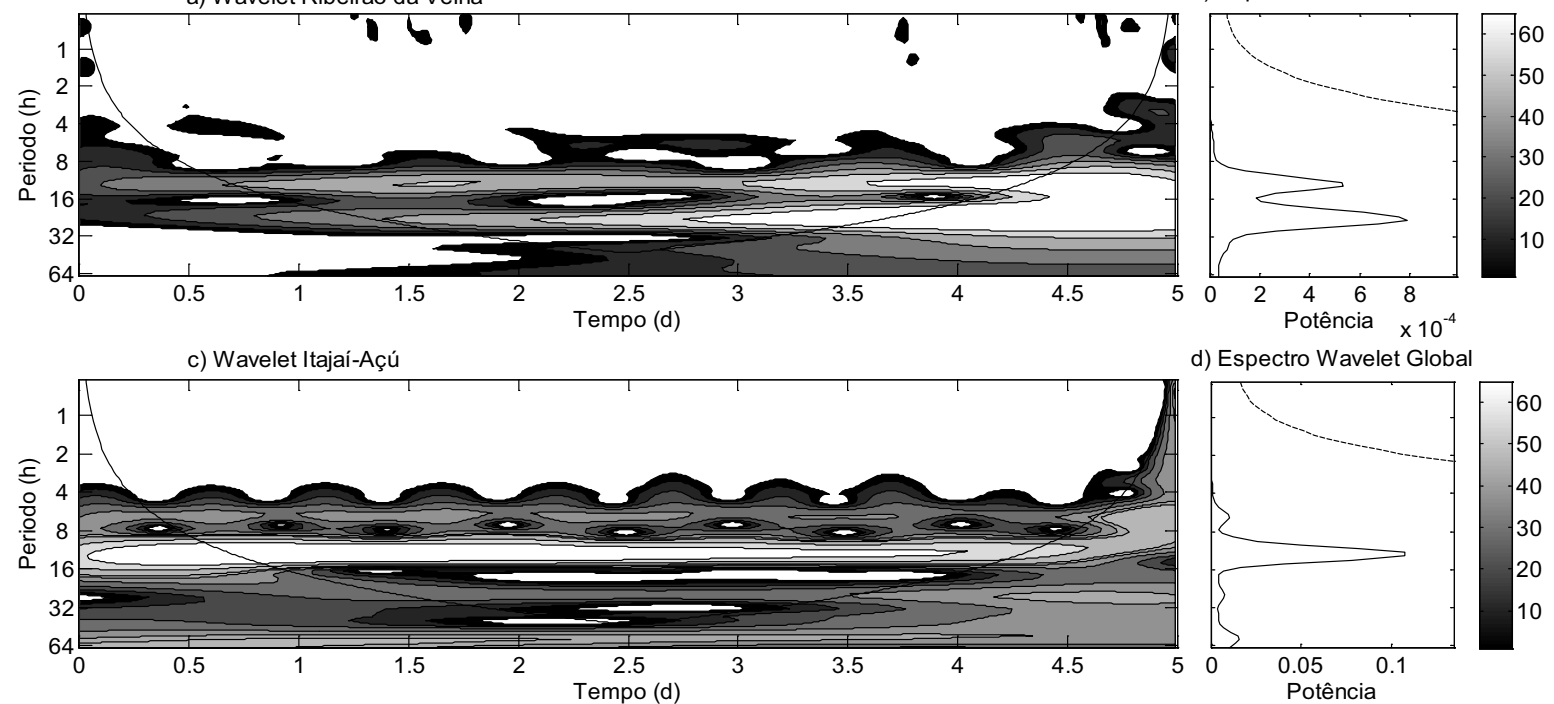
d) Espectro Wavelet Global

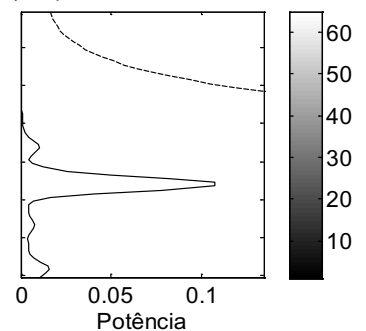

Figura 10 - Análise com transformada wavelet (TW), do rio Itajaí-Açú e do Ribeirão da Velha 
onde os dois sinais variam com a mesma fase (em sincronismo). Ambos os gráficos (XWT e WTC), de acordo com informações presentes em Grinsted et al (2004) se interpretam da seguinte forma:

- As linhas em preto delimitam as áreas onde se tem mais de $95 \%$ de confiança estatística.

- Portanto, em tais regiões, considera-se que a informação de coerência é válida.

- As setas trazem informação sobre a fase entre os dois sinais, da seguinte forma: em fase, apontando para a direita; em contra-fase, apontando para a esquerda; $270^{\circ}$, apontando para cima; $90^{\circ}$, apontando para baixo.

- A escala de cinza determina os níveis de confiança estatística entre os dois sinais, sendo que os tons mais claros correspondem a uma maior significância estatística. Assim, objetivando encontrar mais dados evidenciando a relação entre os dois sinais, efetuou-se a análise XWT (Figura 11) e a análise WTC (Figura 12).

$\mathrm{Na}$ Figura 11 observa-se que ambos os sinais dividem correlação de potência em intervalos em torno de 12 e 24 h. Entretanto com a observação da Figura 12, evidencia-se uma alta coerência de fase entre os dois sinais em intervalos em torno de $12 \mathrm{~h}$. O que comprova a influência da maré de $12 \mathrm{~h}$ encontrada no rio Itajaí-Açú sobre o nível do Ribeirão da Velha.

Ao se visualizar as Figuras 11 e 12 percebe-se que as setas apontam em sua maioria para a direita, o que confirma que estão praticamente em fase (GRINSTED et al 2004).

Ao se confirmar que ambos os sinais apresentam uma forte correlação de potência e fase em torno do intervalo de 12 h utilizou-se essa "janela" de forma a verificar se existe algum "atraso" entre a ocorrência da maré no rio Itajaí-Açú e no Ribeirão da Velha. Para isso fez-se uso da transformada de Fourier FFT, dessa vez para calcular a fase, em graus, entre os

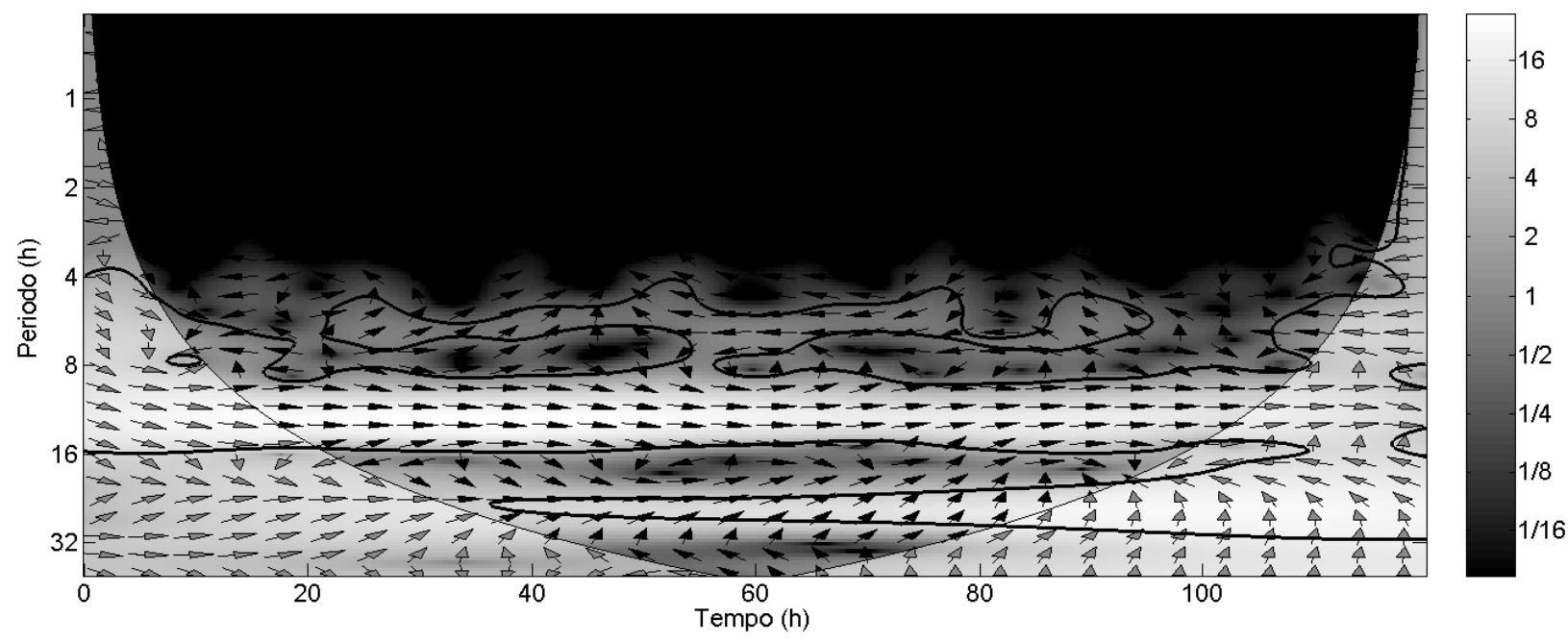

Figura 11 - Análise XWT da transformada Wavelet (TW) para os níveis do rio Itajaí-Açú e do Ribeirão da Velha, para um período de 5 dias

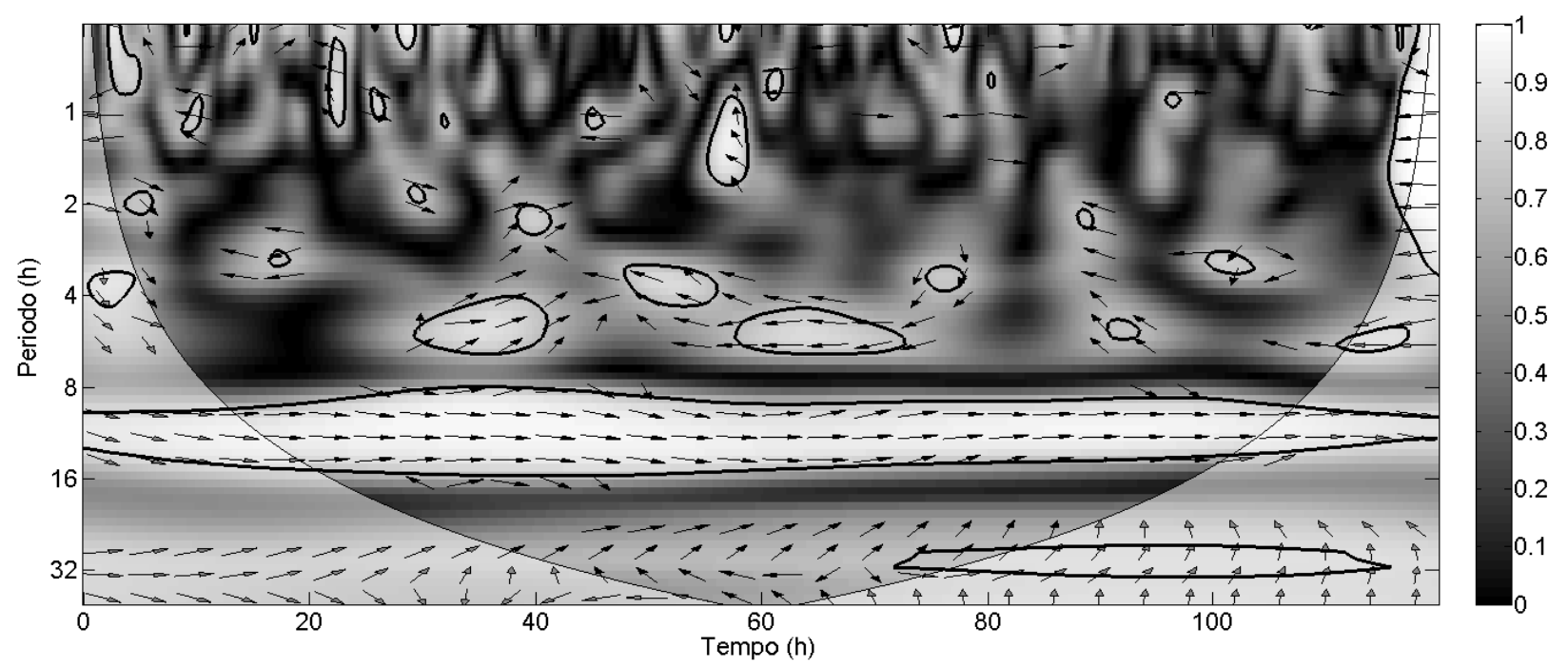

Figura 12 - Análise WTC da transformada Wavelet (TW) para os níveis do rio Itajaí-Açú e do Ribeirão da Velha, para um período de 5 dias 


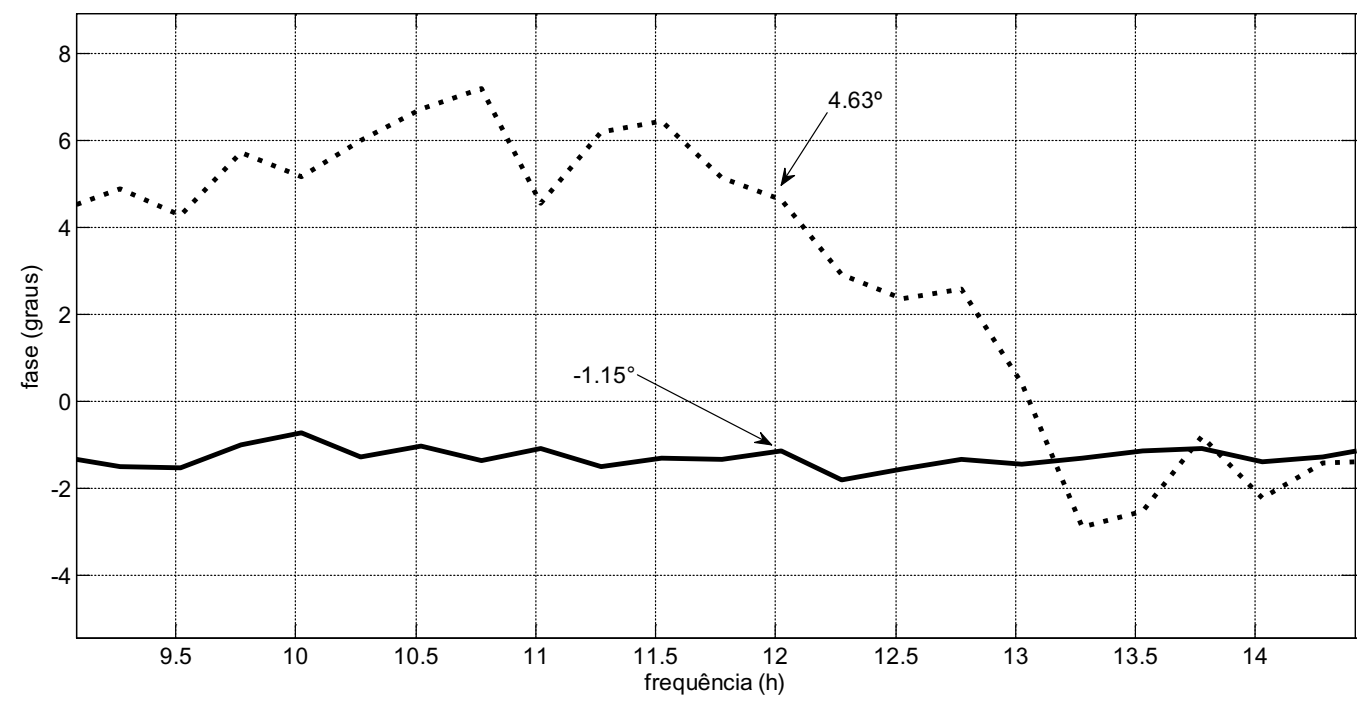

Figura 13 - Análise FFT para a fase dos níveis do rio Itajaí-Açú (em contínuo) e do Ribeirão da Velha (em pontilhado)

dois níveis em torno do período de $12 \mathrm{~h}$, o resultado obtido encontra-se na Figura 13. Observando-se a mesma conclui-se que a diferença de fase entre os dois níveis, em graus, é dada por: $\Delta^{\circ}=4.63^{\circ}-\left(-1.15^{\circ}\right)=5.78^{\circ}$.

Considerando-se que o período da maré no Ribeirão da Velha é de $11.98 \mathrm{~h}$, e que isso equivale a $360^{\circ}$, pode-se concluir que o atraso entre os dois níveis é de 11.54 minutos, ou seja:

$$
\Delta=(5.78 / 360) * 11.98 * 60=11.54 \text { minutos. }
$$

Sabendo-se que a estação de medição do nível do Ribeirão da Velha encontra-se a uma distância de aproximadamente $2.56 \mathrm{~km}$ do rio Itajaí-Açú, concluiu-se que a maré viaja a uma velocidade média aproximada de:

$$
\mathrm{V}=2.56 \mathrm{~km} / 11.54 \text { minutos }=13.31 \mathrm{~km} / \mathrm{h} \text {. }
$$

\section{CONCLUSÕES}

Nesse trabalho realizou-se o estudo da identificação do efeito da maré no Ribeirão da Velha em Blumenau-SC, em períodos de baixa vazão, onde o fenômeno se torna mais perceptível. Também se realizou o estudo da relação da maré no Ribeirão da Velha com a maré do rio Itajaí-Açú.

Para o estudo em questão utilizou-se a transformada wavelet, que tem se tornado uma ferramenta cada vez mais útil e significativa para a interpretação de fenômenos naturais dos mais diversos, em diferentes áreas de estudo. A transformada wavelet constitui uma base matemática robusta que encoraja as atividades de pesquisas científicas que visam a análise de sinais físicos com variabilidade complexa. A utilização desta técnica atualmente é bastante acessível devido ao grande número de rotinas disponíveis em várias linguagens de programação, por exemplo, IDL, Matlab e Fortran.

Verificou-se que, com a utilização do processo de suavização via transformada wavelet, foi possível localizar as características periódicas da maré e confirmar que as mesmas ocorrem durante períodos de baixa vazão. A tradicional análise de Fourier, por outro lado, apesar de apresentar a ocorrência de duas frequências distintas, não fornece qualquer informação sobre o período de ocorrência de tais frequências, tais como, por exemplo, os de baixo fluxo. Por outro lado, quando utilizada a transformada wavelet é explicitamente mostrado no espectro de potência wavelet que o período de ocorrência das marés se evidencia em períodos de baixo fluxo.

Além disso, foi mostrado que é possível determinar de forma simples e bastante rápida a covariabilidade na energia e fase entre duas grandezas físicas, utilizando-se a transformada wavelet, o que levou à comprovação da inter-relação entre as marés que ocorrem no rio principal e no afluente.

\section{REFERÊNCIAS}

BARBOSA, E.B.M.; ROSA, R.R.; VIJAYKUMAR N.L.; BOLZAN, M.J.A.; TOMASELLA, J. Caracterização por Ondaletas de Processos Físicos Não-Lineares. In: Congresso Nacional de Matemática Aplicada e Computacional. São Paulo, SP, Brasil, 2005.

DAUBECHIES, I. “Ten lectures on wavelets": CBMS-NSF conference series in applied mathematics. SIAM Ed, 1992.

DONOHO,D.L.,JOHNSTONE,I.M. IdealSpatial Adaptation via Wavelet shrinkage. Biometrika, v.81, p.425-455, 1994.

DONOHO, D. L.; JOHNSTONE, I. M. Adapting to unknown smoothness via wavelet shrinkage. Journal of American Statistical Association v.90, p.1200-1224, 1995.

GAN, T. Y. Precipitation of Western Canada - Wavelet, scaling, and multifractal analysis and teleconnection 
to large-scale climate anomalies. Proceedings of 15th Hydrotechnical Specialty Conference. Canadian Society of Civil Engineers, Victoria, 2001.

GRINSTED, A., MOORE, J.C., JEVREJEVA, S. Application of the cross wavelet transform and wavelet coherence to geophysical time series, Nonlin. Processes Geophys., v. 11, p. 561-566, 2004. doi:10.5194/npg-11-561-2004

JAY, D. A.; FLINCHEM, E. P. Interaction of fluctuating river flow with a barotropic tide: A demonstration of wavelet tidal analysis methods. Journal of Geophysical Research, v.102, p.5705-5720, 1995.

JAY, D. A.; FLINCHEM; E. P. A comparison of methods for analysis of tidal records containing multi-scale non-tidal background energy. Continental Shelf Research, v.19, p.1695-1732, 1999.

JAY, D. A.; FLINCHEM, E. P. Wavelet transform analyses of non-stationary tidal currents. In: Proceedings of The IEEE Fifth Working Conference on Current Measurement (Edited by A. E. Williams), 1995. p.101-106.

KUMAR, P.; FOUFOULA-GEORGIOU, E. Wavelet analysis for geophysical applications. Rev. Geophys., v.35, p.385- 412, 1997.

LIM, Y.; LYE, L. Wavelet analysis of tideeffected low streamflows series. Journal of Data Science, v.2, p.149-163, 2004.

MORLET, J. G.; ARENS, E. F.; GIARD, D. Wave propogationand sampling theory, 1, Complex signal and scatteringin multilayered media. Geophysics, v.47, n. 2, p.203-221, 1982a.

MORLET, J. G.; ARENS, E. F.; GIARD, D., Wave propagationand sampling theory, 2, Sampling theory and complexwaves. Geophysics, v.47, n. 2, p.222-236, 1982 b.

NASON, G.P.;SACHS, R. V.Wavelets in time series analysis. Phil. Trans. R. Soc. Lond. A. p.357, p.2511-2526, 1999.

PERCIVAL, D.B.; WALDEN, T.A. Wavelet Methods for Time Series Analysis (Cambridge Series in Statistical and Probabilistic Mathematics). Cambridge University Press, 2000.

RONALD R.C. ; DONOHO D.L. Translationinvariant de-noising. In A. Antoniadis and G. Oppenheim, editors, Springer-Verlag. Wavelets and Statistics, v.103, p.125-150. 1995.

SMITH, L.C.; TURCOTT, D.L.; ISACKS, B. L. Stream flow characterization and feature detection using a discrete wavelet transform.
Hydrological Processes, v.12, p.233-249, 1998.

TORRENCE, C.; COMPO, G. P. A practical guide to wavelet analysis. Bulletin of The American Meteorological Society, v.79, p.61-78, 1998.

TRUCCOLO, E.C.; SCHETTINI, C.A.F Marés Astronômicas na baía da babitonga SC. 1999.

WENG, H.; LAU, K.M. Wavelet, period doubling, and timefrequency localization with application to organization of convection over the tropical western Pacific. Jourrnal Atmospheric Sciences, v.51, p. 2523-2541, 1994. 